\title{
Deposition of monomeric C-reactive protein as evidence of localised neurodegenerative diseases
}

\author{
Al Hsinawi $\mathrm{M}^{1}$ and $\mathrm{Al}$ Baradie $\mathrm{R}^{* 2}$ \\ ${ }^{1}$ King Khaled Majmaah Hospital, Al Majmaah, Kingdom of Saudi Arabia \\ ${ }^{2}$ Medical Laboratories Department, College of Applied Medical Sciences, Majmaah University, Al Majmaah, \\ Malek M Al-Hsinawi Director, King Khaled Majmaah Hospital Al Majmaah Kingdom of Saudi Arabia \\ Kingdom of Saudi Arabia
}

\begin{abstract}
Monomeric-C-reactive protein (mCRP) is deposited in significant quantities within the brain parenchyma after stroke. Since we have recently identified a possible role of this protein in supporting neurodegeneration and aberrant vascular development, we identified a small group of post-mortem brain samples from individuals who had AD and on histological examination, evidence of tissue infarction/micro-infarction. Here we show that mCRP deposition is highest in those regions affected by stroke or vascular disruption, and that within those same areas, there is more interaction and co-localization between major classical proteins of neurodegeneration ( $\beta$-amyloid and tau. We hypothesise that vascular disruption and concomitant release of mCRP within the brain tissue could exacerbate ongoing neurological damage via stimulation of neuro-inflammation and from direct consequences of its action on both neuronal and vascular health.
\end{abstract}

\section{KEY WORDS: MONOMERIC C-REACTIVE PROTEIN; STROKE; ALZHEIMER'S DISEASE; NEURODEGENERATION; VASCULAR DEMENTIA}

\section{INTRODUCTION}

We have shown previously that monomeric C-reactive protein (mCRP) was dramatically over-expressed in the brain extracellular matrix (ECM) of patients following acute ischaemic stroke (Slevin, 2010). In addition, mCRP, unlike the native pentameric molecule (pCRP), stimulated aberrant angiogenesis in vitro, induced phosphorylation

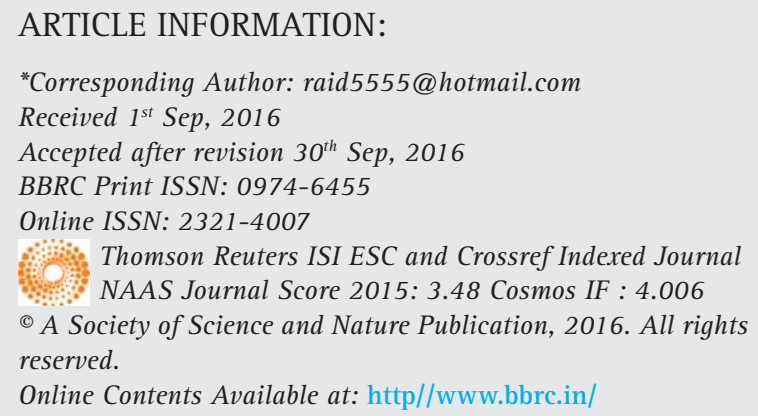

of Tau by neurons and Tau-244-372 aggregation in vitro and following injection into the hippocampus of mice, directly resulted in cognitive and memory decline similar to that seen in a model of Alzheimer's disease (AD) (Tg x 3) ( Slevin, 2015).

mCRP also has been co-localised with CD105 in microvessels suggesting angiogenesis. Phospho-arrays/ Western blotting identified signalling activation in both 
endothelial cells and neurons through p-IRS-1, p-Tau and p-ERK1/2-which was blocked following pre-incubation with mCRP-antibody suggesting that the antibody could have therapeutic potential. mCRP increased vascular monolayer permeability and gap junctions, increased NCAM expression and produced haemorrhagic angiogenesis in mouse matrigel implants and clearly has abnormal effects on the vascular system.

Previously, Strang et al (2012) have demonstrated that $A \beta$ plaques generated in vitro were able to dissociate $\mathrm{pCRP}$ to $\mathrm{mCRP}$, probably produced de-novo within the brain ECM, whilst in vivo, more mCRP was identified within frontal cortex regions in victims of $A D$ than normal control brains suggesting a possible pathological or regulatory role in development of the disease. Cerebrovascular disease, neurovascular dysfunction and cerebral blood flow abnormalities are now recognised as critical influences in the pathophysiological development of $\mathrm{AD}$, damaged, blocked or in patent vessels having a severe effect on the function of local neurovascular units (with approximately $80 \%$ of $\mathrm{AD}$ brains at post-mortem shown to have significant vascular abnormalities), in addition to Vascular dementia, and lowers the threshold for development particularly in younger patients, (Toledo, 2013 and Montagne 2016).

Here, in the present study, we have investigated the possible link between mCRP deposition and localization within the brain and indicators of previous stroke or vascular disruption and dementia.

\section{MATERIAL AND METHODS}

Samples of brain tissue were obtained from the Bristol Brain Bank, where from a cohort of 10 individuals, 3 were identified as having concomitant histological evidence of $\mathrm{AD}$ and stroke (assessed independently and also by our clinical neurologist; Table 1), and a clinical registered history of $\mathrm{AD}$. The $\mathrm{AD}$ cases all had a history of progressive dementia and were selected on the basis of a diagnosis according to CERAD of 'definite AD' (Hyman 2012). AD neuropathological change was considered a sufficient explanation for the dementia in all cases.

Paraffin processed sections from frontal parietal and temporal regions were examined for expression of mCRP and in addition co-staining (double immunohistochemical labelling with VIP-vector kits) for key marker pro- teins of neurodegeneration (p-Tau and $\beta$-amyloid). Particular attention was given to analysis of staining patterns associated with areas of tissue showing evidence of inflammation, vascular damage and regions showing morphological appearance of micro-infarct or other previous stroke.

\section{RESULTS AND DISCUSSION}

The staining pattern revealed that mCRP was almost absent from normal looking regions of brain tissue without signs of neurodegeneration or previous tissue insult. Occasional blood vessels, neurons and glia of brain tissue showed positive mCRP staining. However, mCRP expression increased in the areas demonstrating typical AD pathology (i.e. amyloid deposits and neurofibillary tangles). In these later areas, mCRP was mainly localized to blood vessels and neurons with a smaller expression within glia. Since these areas were not associated with previous infarction, the expression of mCRP is likely to be de novo synthesis similar to that seen by Strang et al [Strang 2012], however, within blood vessel walls, mCRP could infiltrate from the circulation through damaged intimal linings.

mCRP expression was most abundantly present in areas of microinfarction (and adjacent regions) in both grey and white matter, whilst in relatively normal looking areas, expression was weak or none-existent (Figure 1A). mCRP expression was not seen in normal looking regions and hence quantification of the extent of staining was not attempted. This is in line with our previously published observation and hypothesis of a direct link between mCRP deposition and vascular damage in the brain linking it to subsequently and chronically hypo-perfused tissue regions [Slevin 2010].

B-amyloid staining and co-localization: Areas with evidence of previous microinfarctions concomitantly expressed more $\beta$-amyloid. $\beta$-amyloid deposition may increase because of chronic inflammation after stroke associated with chronic cerebrovascular dysfunction, and this could be perpetuated at least in part by the presence of excessive mCRP (Humpel 2011,Thiele, 2014 and McCaulley 2015).

Similarly, build-up of $\beta$-amyloid is known to induce neuro-inflammation directly, thus potentially perpetuating the neurodegenerative consequences (Hyman, 2012).

\begin{tabular}{|l|l|l|l|l|l|}
\hline \multicolumn{6}{|l|}{ Table 1: Patient information } \\
\hline Sample ID & AGE & SEX & Braak-NFT & CERAD & Infarction \\
\hline $496 \mathrm{~T}$ & 84 & F & 2.3 & - & + \\
\hline $691 \mathrm{~F}$ & 83 & $\mathrm{M}$ & 3.4 & 4 & + \\
\hline $697 \mathrm{P}$ & 87 & M & 4.4 & 6 & + \\
\hline
\end{tabular}




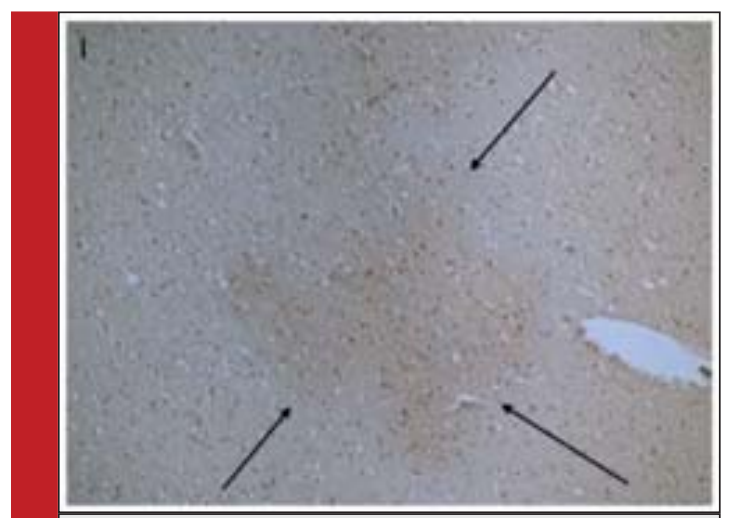

FIGURE 1. Single staining for mCRP-showing increased expression within a micro-infarcted area (496T; arrows; $x$ 100)
Some of the amyloid plaques were close to adjacent blood vessels where mCRP expression was abundant (Figure 1B). Since mCRP is known to stimulate aberrant angiogenesis (Selvin, 2014), this expression could have a potential negative influence on existing or neomicrovessel function and patency contributing to production of a local hypo-perfusive neurodegenerativefriendly environment.

The co-localization between mCRP and $\beta$-amyloid in blood vessels was also mainly seen within areas or adjacent to small infarcts (Figure 1B); however, it was also observed sporadically in other areas of pure tau pathology without clear infarction in the area.

$\beta$-amyloid pathology within existing plaques was mainly separate, with mCRP-positive 'plaque'-like mate-
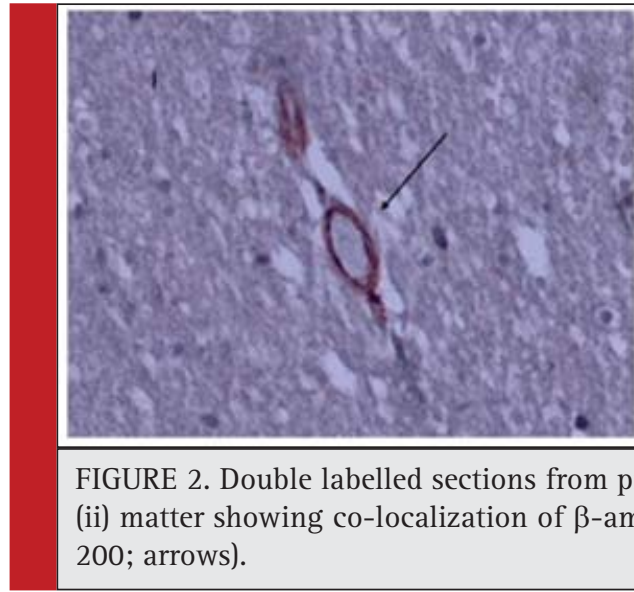
200; arrows).

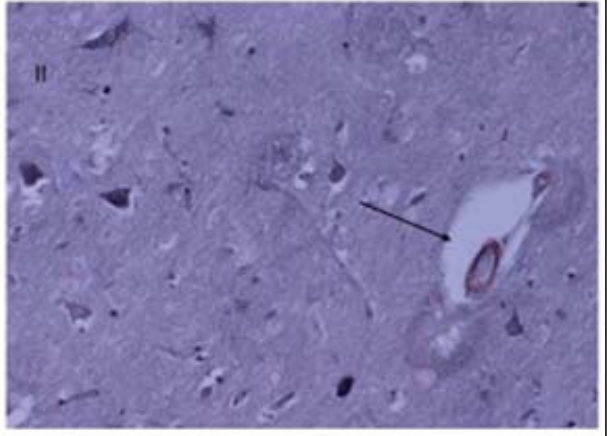

FIGURE 2. Double labelled sections from previously infarcted regions of white (i) and grey (ii) matter showing co-localization of $\beta$-amyloid (red) with mCRP (blue-black) (691F; $x$ rial generally being distinct from regular $\mathrm{AD}$ plaques, in relatively normal looking tissue regions. However, adjacent to previously infarcted regions some of the plaques (approximately 10\% based on counting of $10 \mathrm{x}$ fields of view at $\mathrm{x} 100$ per section of 3 sections) contained a combination of the two proteins (Figure 1C).

p-Tau staining and co-localization: The majority of infarcted areas also exhibited other typical AD pathol-
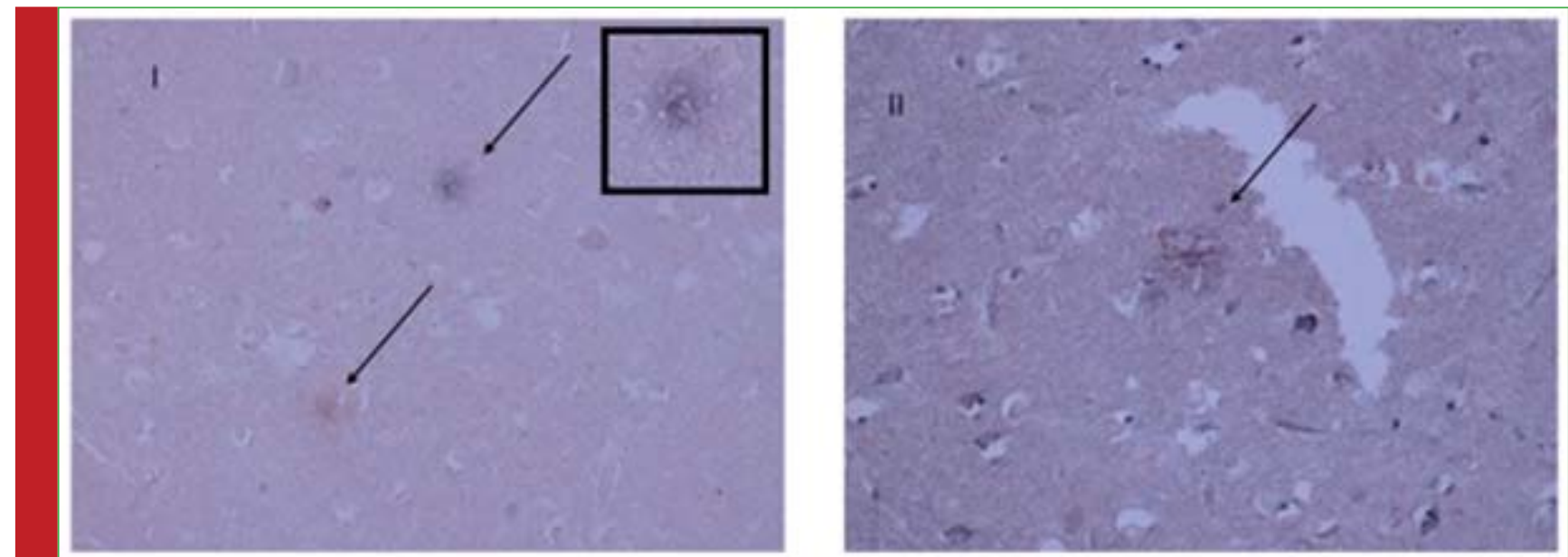

FIGURE 3. Double staining for $\beta$-amyloid and mCRP showing (i) $\beta$-amyloid-positive (red) and mCRP-positive (blue-black) separate plaques in none-infarcted regions (ii) a plaque containing both proteins within a previously infarcted area (arrows). (697P; x 100 (inset x 200) (i) and x 200 (ii)). 

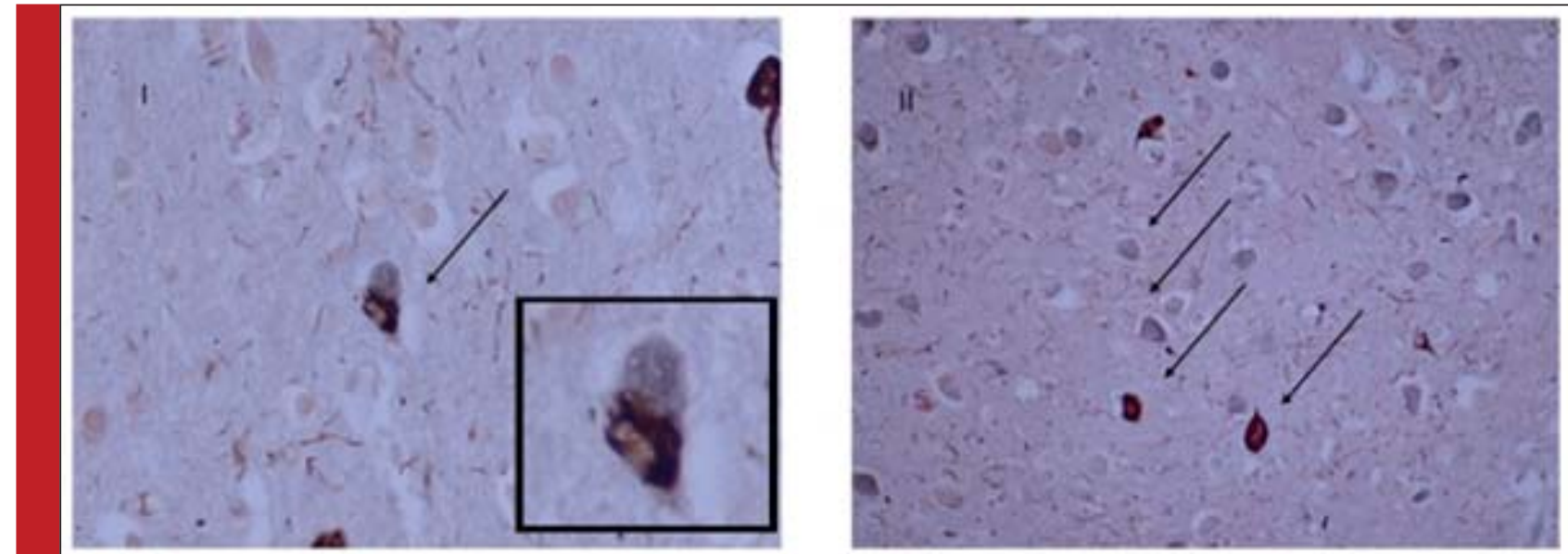

FIGURE 4. Double staining co-localization within micro-infarcted areas of p-tau (red) and mCRP (blue-black) (i) an isolated double-labelled neuron (x 100; inset x 200) and (ii) neurodegenerative grey matter with separately stained neurons for mCRP and $\mathrm{p}$-Tau (arrows).

ogy like Tau deposits. Most of the mCRP co-localized primarily with Tau within neurons in peri-nuclear regions (Figure 1D), although, again, the majority of neurons were either $\mathrm{p}$-Tau or mCRP positive however the number of neurons co-localising with the two proteins was approximately ( $15 \%$ based on counting of $10 \mathrm{x}$ fields of view at $\mathrm{x} 100$ per section of 3 sections). Localization of mCRP with phosphorylated tau in neurons could have physiological significance.

Ourselves and others have demonstrated that mCRP can phosphorylate tau (Ser 202,396) directly in vitro (Selvin 2015; Guo 2015), possibly by a mechanism involving GSK3 $\beta$. It is worthy of note that whilst colocalising, the two proteins $\mathrm{mCRP}$ and $\mathrm{p}$-Tau were present generally at different positions within the neurons.

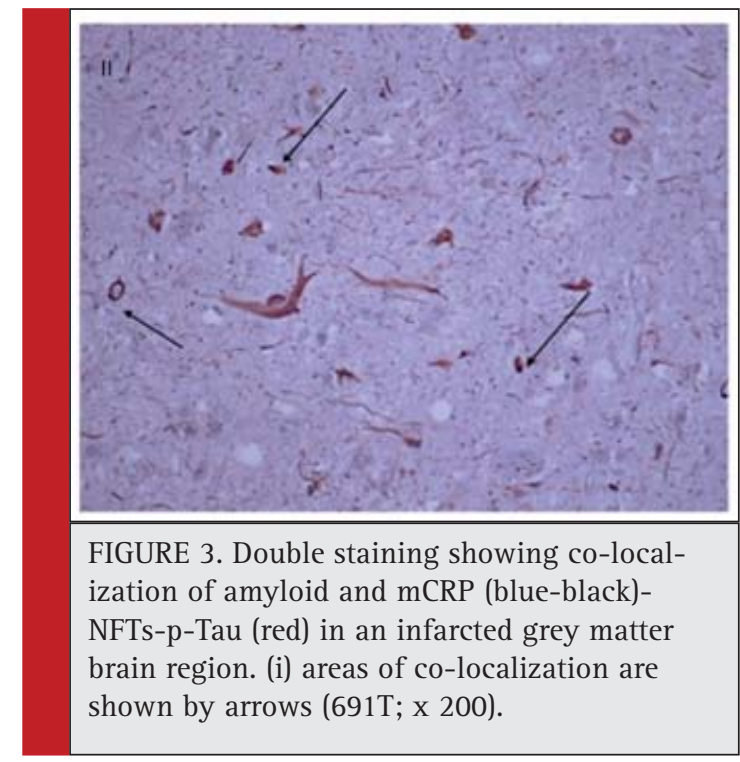

Toxic Tau fibrils (neurofibrillary tangles) were present in some of the most degenerated regions and there was evidence of co-localization of $\mathrm{p}$-Tau with mCRP here also suggesting as our previous work showed that mCRP could contribute or perpetuate to their development (arrows showing dark grey staining) potentially implicating mCRP in its development (Figure E).

Limitations of this 'case study' analysis are clearly from the small numbers of sections and patients reviewed. In addition, the relative timing of events in these patients e.g. infarction versus AD symptoms is not known and so the relative influence of one process on the other is subjective.

Given its strong aberrant biological properties associated with neurodegenerative signalling, vascular modulation and angiogenesis, and its direct perpetuation of inflammatory responses, a clear role for this protein in promoting $\mathrm{AD}$ and $\mathrm{VaD}$ is proposed (Slevin, 2009). The findings shown here strengthen this hypothesis further providing case studies of $\mathrm{AD}$ patients where vascular insults probably linked to a local hypoxic environment appear to be highlighted by a strong deposition of mCRP and concomitant disproportionate evidence of localised neurodegenerative disease. Since the majority of patients who suffer serious stroke go on to develop cognitive decline, lower executive function, and psychomotor processing speed; and over 10\% AD within 5 years (Desmond, 2002 and Mandzia, 2016), further research should study potential mechanisms linking the two conditions with a view to creating protective novel therapeutics.

\section{CONCLUSION}

mCRP is not found in normal-looking brain tissue of non dementia patients, however it is produced and laid down 
in large quantities within the brain following stroke, other brain injury or conditions linked with neuro-inflammation. We hypothesise that vascular disruption and concomitant release of mCRP within the brain tissue could exacerbate ongoing neurological damage via stimulation of neuro-inflammation and from direct consequences of its action on both neuronal and vascular health.

\section{DECLARATIONS-NONE}

Abbreviations used: mCRP-Monomeric C-reactive protein; VaD-Vascular dementia; AD-Alzheimer's disease; ECM-Extra cellular matrix; CERAD-Consortium to establish a registry for Alzheimer's disease.

Ethics approval and consent to participate: Full ethical approval was obtained for the use of tissue samples as detailed by the South West Dementia Brain Bank (SWDBB) and obtained from them.

Consent for publication: Has been obtained by all authors

\section{Availability of data and materials: N/A}

Competing interests: The authors declare they have no competing interests

Funding: This work was supported by the Research Centre of Healthcare Science at Manchester Metropolitan University, and by Sheikh Abdullah bin Abdul Mohsen Al-Tuwaijri project grants within, Majmaah University, Saudi Arabia

\section{ACKNOWLEDGEMENTS}

We would like to thank the South West Dementia Brain Bank (SWDBB) for providing brain tissue for this study. The SWDBB is part of the Brains for Dementia Research programme, jointly funded by Alzheimer's Research UK and Alzheimer's Society and is supported by BRACE (Bristol Research into Alzheimer's and Care of the Elderly) and the Medical Research Council.

This research was supported by Sheikh Abdullah bin Abdul Mohsen Al-Tuwaijri project grants within, Majmaah University, Saudi Arabia. The authors would like to express their gratitude towards Sheikh Abdullah Abdul Mohsen Al-Tuwaijri, Rector Dr. Khalid Saad Al Muqrin for providing the necessary support and assistance in completing this piece of work.

\section{REFERENCES}

Desmond DW, Moroney JT,Sano M, Stern Y (2002), Incidence of Dementia After Ischemic Stroke: Results of a Longitudinal Study. Stroke. (33):2254-2262.

Hyman BT, Phelps CH, Beach TG, Bigo EH, Cairns NJ, Carrillo MC, Dickson DW, Duyckaerts C, Frosch MP, Masliah E, Mirra
SS $<$ Nelson PT, Scneider JA, Thal DR, Thies B, Trojanowski JQ, Vinters HV, Montine TJ (2012) National Institute on AgingAlzheimer's Association guidelines for the neuropathologic assessment of Alzheimer's disease. Alzheimer's Dementia. 8(1): $1-13$.

Guo H, Wang H, Wang C, Cheng Y, Zou Z, Li Y, Wu J, Xu J (2015). C-Reactive Protein Induces Tau Hyperphosphorylation via GSK3 $\beta$ Signaling Pathway in SH-SY5Y Cells. J Mol Neurosci. 56(2):519-27.

Humpel C (2011) Chronic mild cerebrovascular dysfunction for Alzheimer's disease? Exp. Gerentol. 46(4): 225-232.

Mandzia JL, Smith EE, Horton M, Hanly P, Barber PA, Godzwon C, Donaldson E, Asdaghi N, Patel S, Coutts SB (2016). Imaging and Baseline Predictors of Cognitive Performance in Minor Ischemic Stroke and Patients With Transient Ischemic Attack at 90 Days. Stroke. 47(3):726-31.

McCaulley ME, Grush KA (2015) Alzheimer's Disease: Exploring the Role of Inflammation and Implications for Treatment. Int J Alzheimers Dis. Epub 2015 Nov 17.

Montagne A, Nation DA, Pa J, Sweeney MD, Toga AW, Zlokovic BV (2016) Brain imaging of neurovascular dysfunction in Alzheimer's disease. Acta Neuropathol. In press.

Slevin M, and Krupinski J (2009) A role for monomeric C-reactive protein in regulation of angiogenesis, endothelial cell inflammation and thrombus formation in cardiovascular disease? Histol. Histopathol. (2009) (24)11: 1473-1478.

Slevin M, Matou-Nasri S, Turu M, Luque A, Rovira N, Badimon L, Boluda S, Potempa L, Sanfeliu C, de Vera N, Krupinski J (2010). Modified C-reactive protein is expressed by stroke neovessels and is a potent activator of angiogenesis in vitro. Brain Pathol. 20(1):151-65.

Slevin M, Matou S, Zeinolabediny Y Corpas R,Weston R,Liu D,Boras E,Di Napoli M,Petcu E, Sarroca S Popa-Wagner A, Love, S, Font MA, Potempa LA, Al-baradie R, Sanfeliu C, Revilla S, Badimon L, and Krupinski J (2015). Monomeric C-reactive protein-a key molecule driving development of Alzheimer's disease associated with brain ischaemia? Sci. Rep. 2015: 13281.

Strang F, Scheichl A, Chen YC, Wang X, Htun NM, Bassler N, Eisenhardt SU, Habersberger J, Peter K (2012). Amyloid plaques dissociate pentameric to monomeric C-reactive protein: a novel pathomechanism driving cortical inflammation in Alzheimer's disease? Brain Pathol. 22(3):337-46.

Thiele JR, Habersberger J, Braig D, Schmidt Y, Goerendt K, Maurer V, Bannasch H, Scheichl A, Woollard KJ, von Dobschütz E, Kolodgie F, Virmani R, Stark GB, Peter K, Eisenhardt $\mathrm{SU}$ (2014). Dissociation of pentameric to monomeric C-reactive protein localizes and aggravates inflammation: In vivo proof of a powerful proinflammatory mechanism and a new antiinflammatory strategy. Circulation. (1):35-50.

Toledo JB, Arnold SE, Raible K, Brettschneider J, Xie SX, Grossman M, Kukull WA, Trojanowski JQ (2013) Contribution of cerebrovascular disease in autopsy confirmed neurodegenerative disease cases in the National Alzheimer's coordinating centre. Brain. (136):2697-2706. 